International Journal of Theoretical Physics, Vol. 9, No.4(1974),pp. 229-244

\title{
External Inversion, Internal Inversion, and Reflection Invariance
}

\author{
Matej Pavšič \\ Institute J. Stefan, University of Ljubljana, Ljubljana, Yugoslavia \\ Received: 18 June 1973
}

\begin{abstract}
Having in mind that physical systems have different levels of structure we develop the concept of external, internal and total improper Lorentz transformation (space inversion and time reversal). A particle obtained from the ordinary one by the application of internal space inversion or time reversal is generally a different particle. From this point of view the intrinsic parity of a nuclear particle ('elementary particle') is in fact the external intrinsic parity, if we take into account the internal structure of a particle. We show that non-conservation of the external parity does not necessarily imply non-invariance of nature under space inversion. The conventional theory of beta-decay can be corrected by including the internal degrees of freedom to become invariant under total space inversion, though not under the external one.
\end{abstract}

Note: This old paper has recently been subjected to considerable interest due to the increasing exploration of the "exact parity model", "mirror matter" and its possible manifestation in astrophysics. It is not available elsewhere on the Internet, and in the interests of easy access I am posting it on astro-ph. The body of the paper is identical to the published version, except for few minor correction of spelling.

Preseent address: J. Stefan Institute, Jamova 39, SI-1000 Ljubljana, Slovenia E-mail: matej.pavsic@ijs.si 


\section{Introduction}

Though the concept of space inversion is clear to us from the geometrical point of view, we must be careful when applying space inversion to real physical objects. Classical examples show that classical particles have an internal structure which must also be transformed under space inversion. If only positions, translatory and angular momenta are inverted, the transformation is not a complete space inversion, but only a partial one. In the domain of nuclear particles ('elementary particles') we have become accustomed to consider space-time coordinates of particles as one thing and the eventual particle's structure in its internal space as the other thing, independent of space-time. We have believed that when reversing positions, momenta and angular momenta of particles, we have achieved complete space space inversion. Experiments show that the proton has an internal electromagnetic structure. Particles do not differ among themselves only in their space-time properties (spins for instance) but also in other properties, which indicate their internal structure. There is no reason why we should not admit that this internal structure is also due to space-time transformations. We develop the concept of external, internal and total improper Lorentz transformation. We then postulate that each physical theory must be invariant under total improper Lorentz transformation, though not necessarily under an external or internal one.

We apply these ideas to beta-decay. As is well known, the distribution of electrons emitted by oriented $\mathrm{Co}^{60}$ nuclei at the beta-decay is asymmetric with respect to the axis of orientation (Fraunfelder at al., 1957). Polarised electrons are emitted preferentially in one direction and anti-neutrinos in the opposite direction. For electrons and anti-neutrinos have well-defined helicities, the total system has a definite handedness. The mirror picture of the beta-decay is different from the original picture. No such mirror decay was observed, and it was concluded that parity is not conserved in the beta-decay. Hence the weak interaction must contain pseudoscalar terms.

Nature, however, has always appeared to be symmetric in its basic laws, but we have suddenly an unpleasant asymmetry with respect to space inversion. Several attempts have been made in order to save the invariance. One of the proposals was (Salam, 1957) to suggest that every particle has its double which differs from it by its 'handedness'. But, unfortunately, no distinction is made between external and internal space inversion (see also the following text), therefore the theory fails to be convincing for other particles than neutrinos. Our point of view is different from that of Salam. The purpose of this paper is to show how the apparent asymmetrical behavior of the weak processes with respect to space inversion could be explained as the symmetrical behavior by having in mind three types of space inversion: external, internal and the total one.

The symmetric behavior is automatically obtained if we postulate the new kind of particles that are obtained from the ordinary particles by applying to the latter the internal inversion $P_{I}$. Let $a$ (or $a_{+}$) be an ordinary particle, say proton or neutron, etc., and $a_{-}$a particle obtained by internal space inversion (a mirror particle). Two 
particles are related by the internal space inversion $P_{I}$ in the following way

$$
a_{+} \rightarrow a_{-}=P_{I} a_{+}, \quad P_{I} a_{-}=a_{+}
$$

If a particle $a_{+}$has a definite helicity $(\mathbf{s} \cdot \mathbf{p})$, a particle $a_{-}$has the same helicity, but differs from $a_{+}$in its internal structure. What we would like to say is that it is not correct to assume that a mirror image of an elementary particle is generally the same and behaves in the same way in reactions as a particle. But this a priory assumption has been achieved whilst interpreting the asymmetric $\mathrm{Co}^{60}$ beta-decay as the proof for the mirror asymmetry of the weak interaction. By saying that the mirror beta-decay is an impossible process, we tacitly assume that protons or neutrons in the mirror are the same protons or neutrons. There is no experimental evidence for such an assumption. On the contrary, the existence of the anomalous proton or neutron magnetic moments indicates the asymmetric internal structure of two particles. Hence it is possible that the mirror beta-decay exists, but protons or neutrons that decay are mirror protons or mirror neutrons. Therefore, instead of saying that the mirror picture of the processes

$$
\begin{aligned}
& p \rightarrow n+e^{+}+\nu \\
& n \rightarrow p+e^{-}+\bar{\nu}
\end{aligned}
$$

are not possible ones, we can say that the mirror pictures of the processes (1.1) are

$$
\begin{aligned}
& p_{-} \rightarrow n_{-}+e_{-}^{+}+\nu_{-} \\
& n_{-} \rightarrow p_{-}+e_{-}^{-}+\bar{\nu}_{-}
\end{aligned}
$$

with the appropriate directions of spins and momenta. The explanation why process (1.2) have not been observed is that all experiments have been made with protons $p$ and neutrons $n$ and not with mirror protons $p_{-}$and mirror neutrons $n_{-}$. Nuclei of $\mathrm{Co}^{60}$ contain only protons $p$ and neutrons $n$, and no mirror protons $p_{-}$and mirror neutrons $n_{-}$, similarly as they contain no anti-protons and anti-neutrons.

This is the main idea. In the following sections we formulate the concepts of external and internal improper Lorentz transformations, especially external and internal space inversion. External space inversion transforms a particle $a_{+}$(at the position $\mathbf{r}$ with the momentum $\mathbf{p}$ and spin $\mathbf{s}$ into the same particle $a_{+}$(with the same internal structure) at the position $-\mathbf{r}$ with the momentum $-\mathbf{p}$ and the spin $\mathbf{s}$. Internal space inversion transforms a particle $a_{+}$at the position $\mathbf{r}$ with the momentum $\mathbf{p}$ and spin $\mathbf{s}$ and with the left- (right) handed internal structure into the particle $a_{-}$with the same $\mathbf{r}, \mathbf{p}, \mathbf{s}$, but with the opposite, i.e., right- (left) handed, internal structure. Further, we develop the concept of total space inversion which transforms a particle $a_{+}$with given $\mathbf{r}, \mathbf{p}, \mathbf{s}$ and given internal handedness into a particle $a_{-}$with $-\mathbf{r},-\mathbf{p}, \mathbf{s}$, and the opposite internal handedness.

Next we postulate that each interaction should be invariant under total space inversion, though it may not be invariant under external or internal space inversion separately. We then consider the interaction part of the Hamiltonian for the betadecay. This interaction can be modified in such a way that it is invariant under total space inversion, while it is not invariant under partial (external or internal) space 
inversion. The Hamiltonian, so modified, is still the same as the old one for ordinary particles. Finally we discuss further possible consequences that follow from the distinction between external and internal improper Lorentz transformations. Many new theoretical possibilities are revealed.

\section{Distinction between External, Internal and To- tal Improper Lorentz Transformations}

Let us imagine a classical particle, say a stone, with a left-handed shape. Consider the motion of such an internally asymmetric particle with a momentum p. The mirror image of this process is the motion of the right-shaped stone with the momentum $\mathbf{- p}$. If the original left-shaped stone rotates in a certain screw-sense, say left sense, around the axis, defined by the initial direction of motion, then the mirror image of such a process is the motion of the right-shaped stone with the opposite momentum, and spinning in the opposite, i.e. right-screw, sense. We shall name the transformation between the two kinds of motion the space reflection or the total space reflection or inversion. Because rotations in the three-dimensional space do not interest us here, we shall ignore the difference between space reflection and space inversion.

Now imagine such a kind of reflection that ignores the internal asymmetrical structure of the particle. This reflection transforms the left-shaped particle with a momentum $\mathbf{p}$ into the left-shaped particle with the momentum $-\mathbf{p}$; if the particle is spinning in a certain screw-sense, this sense is also reversed under that transformation. We name it the external space reflection or inversion. The definition of the external space inversion is not an artificial one, since if the left-shaped stone, spinning in the left screw-sense, moves with a momentum $\mathbf{p}$ one may always imagine the motion of the same left-shaped stone spinning in the opposite screw-sense and moving with the opposite momentum $-\mathbf{p}$.

The next possibility is to define a transformation which leaves a particle's translational and rotational motion unchanged, but inverses its internal structure with a given handedness into the structure with the opposite handedness. This transformation is the space inversion in the particle's internal system of reference (which is at rest with respect to the particle), which we shall name the internal space inversion. It must be clearly stressed here that the rotation of the particle is with respect to the external frame of reference, hence the internal transformation does not affect the particle's screw-sense rotation.

We shall now use simple symbolism to illustrate the three kinds of space inversion. By definition, space inversion $P$ is a transformation that changes a geometrical point $\mathbf{r}=(x, y, z)$ into the point $-\mathbf{r}$. If the point $\mathbf{r}$ moves with velocity $\mathbf{v}$, its velocity is changed into $\mathbf{- v}$ under $P$. Hence

$$
\begin{aligned}
P: & \mathbf{r} \rightarrow \mathbf{r}^{\prime}=-\mathbf{r} \\
& \mathbf{v} \rightarrow \mathbf{v}^{\prime}=-\mathbf{v}
\end{aligned}
$$


A geometrical object $A(\mathbf{r})$ (a tensor) defined at the position $\mathbf{r}$ transforms under the space inversion after the well-known rules for transformation of tensors.

Let us consider a classical particle which is a physical object, not a geometrical point. It has a finite size and has both translational and rotational degrees of freedom. Its state of motion is defined by coordinates $\mathbf{r}$ of its mass centre, momentum $\mathbf{p}$ and intrinsic angular momentum $\mathbf{s}$ (pure rotation or spinning of the particle) as functions of time. Under space inversion the three quantities transform as

$$
\begin{array}{rll}
P: & \mathbf{r} \rightarrow \mathbf{r}^{\prime}=-\mathbf{r} & \text { vector } \\
& \mathbf{p} \rightarrow \mathbf{p}^{\prime}=-\mathbf{p} & \text { vector } \\
& \mathbf{s} \rightarrow \mathbf{s}^{\prime}=\mathbf{s} & \text { pseudovector }
\end{array}
$$

The state of a particle is, however, not completely characterized by the quantities $\mathbf{r}$, $\mathbf{p}$, and $\mathbf{s}$, for the full description we must also take into account the particle's internal structure. This structure generally transforms under space inversion, which means that in the internal coordinate frame each point $a$ with coordinates $\vec{\xi}=\left(\xi_{x}, \xi_{y}, \xi_{z}\right)$ is transformed into the point $a^{\prime}$ with coordinates $-\vec{\xi}$ :

$$
\begin{array}{ll}
P: \quad & a \rightarrow a^{\prime}=P a \\
& \vec{\xi} \rightarrow \vec{\xi}^{\prime}=-\vec{\xi}
\end{array}
$$

In the laboratory coordinate system the same point $a$ has the coordinates $\mathbf{r}+\vec{\xi}$. By bearing in mind the upper transformation performed in the internal frame, we can construct the following types of transformation performed in the laboratory system:

(a) total space inversion

$$
\begin{aligned}
P_{T}: \quad & a \rightarrow a^{\prime \prime}=P_{T} a \\
& \mathbf{r}+\vec{\xi} \rightarrow \mathbf{r}^{\prime \prime}+\vec{\xi}^{\prime \prime}=-\mathbf{r}-\vec{\xi}
\end{aligned}
$$

(b) external space inversion

$$
\begin{aligned}
P_{E}: \quad & a \rightarrow a^{\prime \prime \prime}=P_{E} a \\
& \mathbf{r}+\vec{\xi} \rightarrow \mathbf{r}^{\prime \prime \prime}+\vec{\xi}^{\prime \prime \prime}=-\mathbf{r}+\vec{\xi}
\end{aligned}
$$

(c) internal space inversion

$$
\begin{aligned}
P_{I}: \quad & a \rightarrow a^{\prime}=P_{I} a \\
& \mathbf{r}+\vec{\xi} \rightarrow \mathbf{r}^{\prime}+\vec{\xi}^{\prime}=-\mathbf{r}-\vec{\xi}
\end{aligned}
$$

The last two transformations are only partial space inversions in such a sense that either $\mathbf{r}$ is reversed and $\vec{\xi}$ left unchanged (b), or $\mathbf{r}$ is left unchanged and $\vec{\xi}$ is reversed (c). Obviously

$$
P_{T}=P_{E} P_{I}=P_{I} P_{E}
$$

Here $\mathbf{r}$ is the external coordinate, describing the particle's position in a fixed (laboratory) frame, and $\vec{\xi}$ is a coordinate associated with the internal structure of the particle. Since the internal structure is not determined by the single coordinate 
$\vec{\xi}$ but (roughly speaking) with the distribution of matter within the particle, we shall use the symbol $\alpha$ in order to denote the parameter of internal inversion. $\alpha$ has two discrete values; +1 and -1 . They belong to two opposite states of internal inversion. In other words, $\alpha$ denotes a set of all internal degrees of freedom which suffer the internal space inversion. The total state of motion of a particle with respect to the laboratory system is described by parameters $\mathbf{r}, \mathbf{p}, \mathbf{s}, \alpha$. The space inversion yields:

$$
\begin{array}{ll}
P_{T}: & (\mathbf{r}, \mathbf{p}, \mathbf{s}, \alpha) \rightarrow\left(\mathbf{r}^{\prime}, \mathbf{p}^{\prime}, \mathbf{s}^{\prime}, \alpha^{\prime}\right)=(-\mathbf{r},-\mathbf{p}, \mathbf{s},-\alpha) \\
P_{I}: & (\mathbf{r}, \mathbf{p}, \mathbf{s}, \alpha) \rightarrow\left(\mathbf{r}^{\prime}, \mathbf{p}^{\prime}, \mathbf{s}^{\prime}, \alpha^{\prime}\right)=(\mathbf{r}, \mathbf{p}, \mathbf{s},-\alpha) \\
P_{E}: & (\mathbf{r}, \mathbf{p}, \mathbf{s}, \alpha) \rightarrow\left(\mathbf{r}^{\prime}, \mathbf{p}^{\prime}, \mathbf{s}^{\prime}, \alpha^{\prime}\right)=(-\mathbf{r},-\mathbf{p}, \mathbf{s}, \alpha)
\end{array}
$$

By the following example we make the concept of the transformations $P_{T}, P_{E}, P_{I}$ as clear as possible. An artist takes several pieces of optically active substance. Each piece has asymmetric shape. He then makes a structure that consists of these pieces. Next, he reverses the positions of all the pieces in the structure and so obtains another structure which is a partial mirror image of the previous structure; this transformation is the external space inversion. He then replaces all the pieces in the last structure with the pieces that have the shape of the opposite handedness. If he stops his consideration at this point he will state that the last transformation is the total space inversion. However, he can go further. If the pieces consist of the optically active substance with the left screw-sense he can replace them by pieces with the right screw-sense. Moreover, he may also distinguish the reflection of the shape from the reflection of the positions of all the molecules within the piece. $\mathrm{He}$ can proceed to a finer and finer level of structure.

The example above shows that though the concept of pure geometrical space inversion (i.e., the inversion of positions of the separate geometrical points and the appropriate geometrical objects - tensors - defined at these points) is clear to us, we must be careful when applying the space inversion to the real physical object. It is true that partial - for instance the external- space inversion is in fact not the real space inversion in the geometrical sense, because, if we speak about the partial space inversion, we are aware of the further finer levels of complexity in the observed system. But, dynamically, whatever kind of physical system we try to transform by space inversion, at last we necessarily arrive at the level of complexity where our knowledge about a more detailed structure comes to an end. At this level we do not distinguish between external and total space inversion. In every day language we speak about the space inversion of a physical system if all degrees of freedom that we are able to control are reversed. The physical system is usually composed of sub-systems at different levels of complexity. Such a physical system can be transformed also by partial space inversion - external or internal. Partial space inversion is intimately connected with our knowledge about the levels of complexity of the system.

Similarly, as in the case of space inversion, we must also distinguish between total, internal and external time reversal. We denote them $T_{T}, T_{I}$ and $T_{E}$, respectively. They transform the physical system in the following way: 


$$
\begin{array}{ll}
T_{T}: & (\mathbf{r}, t, \mathbf{p}, \mathbf{s}, \tau) \rightarrow\left(\mathbf{r}^{\prime}, t^{\prime}, \mathbf{p}^{\prime}, \mathbf{s}^{\prime}, \tau^{\prime}\right)=(\mathbf{r},-t,-\mathbf{p},-\mathbf{s},-\tau) \\
T_{I}: & (\mathbf{r}, t, \mathbf{p}, \mathbf{s}, \tau) \rightarrow\left(\mathbf{r}^{\prime}, t^{\prime}, \mathbf{p}^{\prime}, \mathbf{s}^{\prime}, \tau^{\prime}\right)=(\mathbf{r}, t, \mathbf{p}, \mathbf{s},-\tau) \\
T_{E}: & (\mathbf{r}, t, \mathbf{p}, \mathbf{s}, \tau) \rightarrow\left(\mathbf{r}^{\prime}, t^{\prime}, \mathbf{p}^{\prime}, \mathbf{s}^{\prime}, \tau^{\prime}\right)=(\mathbf{r},-t,-\mathbf{p},-\mathbf{s}, \tau)
\end{array}
$$

where $\tau$ if the parameter that characterizes the internal time. The philosophy is analogous here as in the case of space inversion. The combination of all $P$ and $T$ types of transformations are also possible, but the consideration of these possibilities goes beyond the purpose of the present paper.

The quantum system in the state $\phi(\mathbf{r}, t, \mathbf{s}, \alpha, \tau)$ transforms under the improper Lorentz transformation as

$$
\phi(\mathbf{r}, t, \mathbf{s}, \alpha, \tau) \rightarrow \phi\left(\mathbf{r}^{\prime}, t^{\prime}, \mathbf{s}^{\prime}, \alpha^{\prime}, \tau^{\prime}\right)
$$

where the transformed coordinates $\mathbf{r}, t, \mathbf{s}, \alpha, \tau$ are replaced according to (2.1) or (2.2). For instance, the description of an elementary particle by state vector $\phi(\mathbf{r}, t, \mathbf{s})$ is incomplete. The incompleteness can be seen just from the fact that for the particle dynamics, as we have constructed, discrete Lorentz transformations do not form a symmetry group for all interactions. The interaction that governs the beta-decay is not invariant under space inversion. We shall show later that this non-invariance can be ascribed to the incomplete description of the system by the state vector $\phi(\mathbf{r}, t, \mathbf{s})$. By including the internal degrees of freedom $\alpha$ we can reformulate the dynamics of the beta-decay so that it becomes invariant under total space inversion. This reformulation implies the existence of mirror particles, i.e., particles with all other quantum numbers as spin, charge, mass, etc., unchanged, but with the reversed quantum number $\alpha$ - the parameter of internal inversion. Because we are now inclined to consider invariance principles as first principles it seems natural for us to accept the proposal as a serious possibility, which has to be checked experimentally.

\section{Invariance of Interactions Under the Total Space Inversion}

The main postulate and the starting point of this article is that each physical theory must be invariant under the full group of Lorentz transformations with the improper transformations included. If observations show that a class of phenomena exists, which transformed by any Lorentz transformations are not the possible phenomena, it means that our theory is not invariant under those transformations. We can adopt two points of view. First, that our theory is correct and that nature really possesses asymmetry; and second, that our theory is incomplete, while nature is symmetric. History teaches us that the second point of view has always proved to be more convenient. Thus, in the situation where our theory appears to be noninvariant under any of the Lorentz transformations, we must admit that we have not yet obtained complete knowledge about the observed phenomena. The theory must be completed by including the internal degrees of freedom of particles involved in the observed phenomena. While studying the behavior of processes under the space 
inversion, the internal handedness of particles must be taken into account in those cases in which the inversed process would be otherwise an impossible process.

The state of a system is represented by the state vector $\phi(x, \alpha)$, where $\alpha$ is the set of all internal degrees of freedom which are due to space inversion and $x=(\mathbf{r}, t)$. We shall assume that $x$ and $\alpha$ are independent, which gives $\phi(x, \alpha)=$ $\phi(x) \varphi(\alpha)$. Parameter $x$ is included in order to demonstrate that the state vector $\phi(x)$ is completely represented by the field $\phi(x)$ defined over $x$. The symbol $\phi(x, \alpha)$ denotes the state vector which is not completely represented by the field $\phi(x)$ and additional degrees of freedom $\alpha$ have to be taken into account. Parameter $\mathbf{s}$, denoting spin, is omitted. In a given representation, spin is determined by the type of field (scalar, vector). The transformations are

$$
\begin{gathered}
P_{T} \phi(x, \alpha)=\phi^{\prime}\left(x^{\prime}, \alpha^{\prime}\right)=\phi^{\prime}(-\mathbf{r}, t,-\alpha)=\phi^{\prime}(-\mathbf{r}, t) \varphi^{\prime}(-\alpha) \\
P_{E} \phi(x, \alpha)=\phi^{\prime}\left(x^{\prime}, \alpha^{\prime}\right)=\phi^{\prime}(-\mathbf{r}, t, \alpha)=\phi^{\prime}(-\mathbf{r}, t) \varphi^{\prime}(\alpha) \\
P_{I} \phi(x, \alpha)=\phi^{\prime}\left(x^{\prime}, \alpha^{\prime}\right)=\phi^{\prime}(\mathbf{r}, t,-\alpha)=\phi^{\prime}(\mathbf{r}, t) \varphi^{\prime}(-\alpha)
\end{gathered}
$$

We postulate that the Hamiltonian $H$ is invariant under total space inversion $P_{T}$, which means that both operators commute

$$
\left[P_{T}, H\right]=0
$$

From $P_{T}=P_{E} P_{I}=P_{I} P_{E}, P_{E} \neq 0, P_{I} \neq 0$ and from

$$
\left[P_{T}, H\right]=\left[P_{E} P_{I}, H\right]=P_{E}\left[P_{I}, H\right]+\left[P_{E}, H\right] P_{I}=0
$$

follows either

$$
\left[P_{E}, H\right]=0 \quad \text { and } \quad\left[P_{I}, H\right]=0
$$

or

$$
\left[P_{E}, H\right] \neq 0 \quad \text { and } \quad\left[P_{I}, H\right] \neq 0
$$

Equation (3.1) implies that if $\phi(x, \alpha)$ is an eigenstate of $H$ with the energy $E$, then $P_{T} \phi(x, \alpha)$ is an eigenstate of the same $H$ with the same energy. Generally $\phi(x, \alpha)$ and $P_{T} \phi(x, \alpha)$ are not the same, and there is a degeneracy with respect to total space inversion.

Operator $P_{E}$ either commutes with $H$ or does not commute. In the first case the states $\phi(x, \alpha)$ and $P_{E} \phi(x, \alpha)$ are both eigenstates of $H$ with the same energy $E$. We do not observe any degeneracy due to external space inversion, hence both $\phi(x, \alpha)$ and $P_{E} \phi(x, \alpha)$ represent the same state. Following well-known procedure we conclude that the state $\phi(x, \alpha)$ has the definite parity which is conserved under the interaction $H$. In the second case, parity is not a good quantum number and is not conserved. We stress explicitly that parity relates to the external part $\phi(x)$ of the total state vector $\phi(x) \varphi(\alpha)$ and the external parity operator $P_{E}$.

Whether the external parity is conserved or not depends on the kind of interaction $H$. The strong interaction, for instance, conserves parity to a high degree of precision, while the weak interaction violates the external parity. We would like to 
show that the non-conservation of external parity does not necessarily imply the noninvariance of nature under space inversion. The conventional theory of beta-decay is not invariant under space inversion, and it can be improved to become invariant under space inversion by including the internal degrees of freedom. However, this is only a theoretical possibility, to be proved or disproved by experiment.

The proposed properties of the strong and the weak interactions will now be described.

\section{The strong Interaction}

The strong interaction is invariant under the transformation $P_{E}$ :

$$
\left[P_{E}, H\right]=0
$$

From (3.1) it follows that also $\left[P_{I}, H\right]=0$. As is experimentally established, there is no degeneracy with respect to the external space inversion and we have

$$
\begin{gathered}
P_{E} \phi(\mathbf{r}, t) \varphi(\alpha)=\phi^{\prime}(-\mathbf{r}, t) \varphi(\alpha)=\xi_{E} \phi(\mathbf{r}, t) \varphi(\alpha) \\
P_{E}^{2} \phi(\mathbf{r}, t) \varphi(\alpha)=P_{E} \xi_{E} \phi(\mathbf{r}, t) \varphi(\alpha)=\xi_{E}^{2} \phi(\mathbf{r}, t) \varphi(\alpha)=\phi(\mathbf{r}, t) \varphi(\alpha)
\end{gathered}
$$

therefore

$$
\xi_{E}= \pm 1
$$

$\xi_{E}$ is the external intrinsic parity of the state $\phi(\mathbf{r}, t) \varphi(\alpha)$. It is a good quantum number. For instance, different particles have different external intrinsic parities.

With respect to the internal space inversion $P_{I}$ we have to admit two possibilities: either a degeneracy or a definite internal parity. The possibility of the definite internal parity, i.e. the identity of states $\phi(x) \varphi(\alpha)$ and $P_{I} \phi(x) \varphi(\alpha)$, is excluded for nucleons, because we postulate that $\left[P_{T}, H\right]=0$ for all interactions. In the case of the weak interaction this would not be fulfilled because we could not introduce mirror nucleons. Hence there is a degeneracy: $\phi(x) \varphi(\alpha)$ and $P_{I} \phi(x) \varphi(\alpha)$ represent two different states, both eigenstates of the same Hamiltonian. If $\phi(x) \varphi(\alpha)$ is the state of a nucleon $p$ (denoted also as $p_{+}$), then $P_{I} \phi(x) \varphi(\alpha)=\phi(x) \varphi^{\prime}(-\alpha)$ is the state of a mirror nucleon $p_{-}$.

In order to explain why both degenerate states do not occur in the nucleus, we have to assume that the strong interaction is strong enough to yield bound states only if it works on particles of the same kind, otherwise it gives no bound states. The scheme for the two-body strong interaction is

$$
\begin{array}{lll}
\alpha_{1}=1, & \alpha_{2}=1 & \text { strong attractive force } \\
\alpha_{1}=1, & \alpha_{2}=-1 & \text { weak attractive or repulsive force } \\
\alpha_{1}=-1, & \alpha_{2}=1 & \text { weak attractive or repulsive force } \\
\alpha_{1}=-1, & \alpha_{2}=-1 & \text { strong attractive force }
\end{array}
$$

The natural conclusion, therefor, is if there are nuclei with $p_{+}$and $n_{+}$there must also be nuclei with $p_{-}$and $n_{-}$. Are such nuclei in the ordinary matter on the earth 
in small concentrations (like isotopes) or in large concentrations? Possibly mirror nucleons do not constitute ordinary matter like anti-nucleons, and could be found only under extreme conditions, for instance at high energies, in cosmic rays or in the other parts of the Universe. We have not sufficient information from experiment to decide which possibility holds, if either.

\section{The Weak Interaction}

In the conventional picture of beta-decay is governed by the Hamiltonian which is not invariant under space inversion. But as we have shown it is necessary to distinguish among external, internal and total space inversion. Each interaction is, after our postulate, invariant under total space inversion, though not necessarily under partial space inversion. The Hamiltonain for the beta-decay (Lee \& Yang, 1957) can be modified in such a way that it is invariant under total space inversion.

$$
H_{\mathrm{int}}=\frac{1}{\sqrt{2}} \sum_{i} G_{i}\left[\bar{\psi}_{p}(x, \alpha) \Gamma_{i} \psi_{n}(x, \alpha)\right]\left[\bar{\psi}_{e}(x, \alpha) \Gamma_{i}\left(1+\eta \gamma_{5}\right) \psi_{\nu}(x, \alpha)\right]
$$

$\psi(x, \alpha)$ is a field defined formally over the external space-time coordinate $x$ and the internal parameter $\alpha$. Detailed knowledge of the field is not necessary for the present considerations. We only require that $x$ and $\alpha$ are independent and that $\psi(x, \alpha)$ is a solution of the Dirac equation. We can the write formally

$$
\begin{aligned}
\psi(x, \alpha) & =\psi(x) \varphi(\alpha) \\
\bar{\psi}(x, \alpha) & =\varphi^{\dagger}(\alpha) \bar{\psi}(x)
\end{aligned}
$$

where $\psi(x)$ is a Dirac field and $\varphi(\alpha)$ a field associated with the internal state. Parameter $\eta$ in equation (3.2) depends upon the values of $\alpha$ of individual particles involved in the beta-decay.

$$
\eta=\left\{\begin{aligned}
1 & \text { for } \quad \alpha_{p}=\alpha_{n}=\alpha_{e}=\alpha_{\nu}=1 \\
-1 & \text { for } \alpha_{p}=\alpha_{n}=\alpha_{e}=\alpha_{\nu}=-1
\end{aligned}\right.
$$

If one of the $\alpha^{\prime} s$ differs from the others there is no weak interaction, or it is qualitatively different. For positive $\eta$ the weak interaction produces the right-handed beta-decay, and for negative $\eta$ the left-handed beta-decay, where signatures rightleft are chosen arbitrarily. The situation has its classical analog in a gun, with a spiral trace inside the barrel. If a bullet has a spiral on its surface corresponding to the spiral inside the barrel, one obtains the 'interaction' which causes the fired bullet to rotate in a certain screw-sense. If the spiral of the bullet does not fit the gun's spiral, there is no such screw-sense interaction and the missile will not leave the barrel.

Inserting (3.3) into (3.2) the Hamiltonian becomes

$$
H_{\mathrm{int}}=\sum_{i} \frac{G_{i}}{\sqrt{(} 2)}\left[\varphi_{p}^{\dagger}(\alpha) \bar{\psi}_{p}(x) \Gamma_{i} \psi_{n}(x) \varphi_{n}(\alpha)\right]\left[\varphi_{e}^{\dagger}(\alpha) \bar{\psi}_{e}(x) \Gamma_{i}\left(1+\eta \gamma_{5}\right) \psi_{\nu}(x) \varphi_{\nu}(\alpha)\right]
$$


This can be written in a more simple form (see for instance Muirhead, 1968):

$$
\begin{aligned}
H_{\text {int }} & =\frac{G_{V}}{\sqrt{2}}\left[\varphi_{p}^{\dagger}(\alpha) \bar{\psi}_{p}(x) \gamma_{\lambda} \psi_{n}(x) \varphi_{n}(\alpha)\right]\left[\varphi_{e}^{\dagger}(\alpha) \bar{\psi}_{e}(x) \gamma_{\lambda}\left(1+\eta \gamma_{5}\right) \psi_{\nu}(x) \varphi_{\nu}(\alpha)\right] \\
& +\frac{G_{A}}{\sqrt{2}}\left[\varphi_{p}^{\dagger}(\alpha) \bar{\psi}_{p}(x) i \gamma_{\lambda} \gamma_{5} \psi_{n}(x) \varphi_{n}(\alpha)\right]\left[\varphi_{e}^{\dagger}(\alpha) \bar{\psi}_{e}(x) i \gamma \lambda \gamma_{5}\left(1+\eta \gamma_{5}\right) \psi_{\nu}(x) \varphi_{\nu}(\alpha)\right]
\end{aligned}
$$

where $G_{V}$ and $G_{A}$ are vector and axial-vector coupling constants, respectively. The total space inversion gives

$$
\begin{gathered}
\psi(x, \alpha) \rightarrow \psi^{\prime}\left(x^{\prime}, \alpha^{\prime}\right)=P_{T} \psi(x, \alpha)=P_{E} \psi(x) P_{I} \varphi(\alpha)=\left\{\begin{array}{l} 
\pm \gamma_{4} \psi(x) P_{I} \varphi(\alpha) \\
\pm i \gamma_{4} \psi(x) P_{I} \varphi(\alpha)
\end{array}\right. \\
\bar{\psi}(x, \alpha) \rightarrow \bar{\psi}^{\prime}\left(x^{\prime}, \alpha^{\prime}\right)=\varphi^{\dagger \dagger}\left(\alpha^{\prime}\right) \psi^{\prime \dagger}\left(x^{\prime}\right) \gamma_{4}=\varphi^{\dagger}(\alpha) P_{I}^{\dagger} \psi^{\dagger}(x) P_{E}^{\dagger} \gamma_{4} \\
=\left\{\begin{array}{l}
\varphi^{\dagger}(\alpha) P_{I}^{\dagger} \psi^{\dagger}\left( \pm \gamma_{4}\right) \gamma_{4} \\
\varphi^{\dagger}(\alpha) P_{I}^{\dagger} \psi^{\dagger}\left(\mp i \gamma_{4}\right) \gamma_{4}
\end{array}\right.
\end{gathered}
$$

Here $P_{E}$ is identical with the operator $S$ that performs the space inversion on the Dirac field. It is equal to $\pm \gamma_{4}$ or $\pm i \gamma_{4}$ for the real or the imaginary parity class, respectively. The following relations are satisfied:

(I) Real parity class

$$
\begin{gathered}
P_{E} P_{E}=1, \quad P_{E}^{\dagger} P_{E}=1 \\
P_{E}=P_{E}^{-1}= \pm \gamma_{4}=P_{E}^{\dagger}
\end{gathered}
$$

(II) Imaginary parity class

$$
\begin{gathered}
P_{E} P_{E}=-1, \quad P_{E}^{\dagger} P_{E}=1 \\
P_{E}=-P_{E}^{-1}= \pm i \gamma_{4}=-P_{E}^{\dagger}
\end{gathered}
$$

We shall assume that operator $P_{I}$ is also unitary

$$
P_{I} P_{I}^{\dagger}=P_{I}^{\dagger} P_{I}=1
$$

Hamiltonian (3.4) consists of the terms which are transformed as

$$
\begin{aligned}
V^{\lambda}=\varphi^{\dagger}(\alpha)[ & \left.\bar{\psi}(x) \gamma_{\lambda} \psi(x)\right] \varphi(\alpha) \\
\rightarrow V^{\prime \lambda} & =\varphi^{\prime} \dagger\left(\alpha^{\prime}\right)\left[\bar{\psi}^{\prime}\left(x^{\prime}\right) \gamma_{\lambda} \psi^{\prime}\left(x^{\prime}\right)\right] \varphi^{\prime}\left(\alpha^{\prime}\right) \\
& =\varphi^{\dagger}(\alpha) P_{I}^{\dagger}\left[\psi^{\dagger}(x) P_{E}^{\dagger} \gamma_{4} \gamma_{\lambda} P_{E} \psi(x)\right] P_{I} \varphi(\alpha) \\
& =\varphi^{\dagger}(\alpha) P_{I}^{\dagger}\left[a_{\lambda \alpha} \bar{\psi}(x) \gamma_{\alpha} \psi(x)\right] P_{I} \varphi(\alpha) \\
& =\varphi^{\dagger}(\alpha)\left[a_{\lambda \alpha} \bar{\psi}(x) \gamma_{\alpha} \psi(x)\right] \varphi(\alpha) \\
& =a_{\lambda \alpha} V^{\alpha}
\end{aligned}
$$

where

$$
\left(a_{\lambda \alpha}\right)=\left(\begin{array}{cccc}
-1 & 0 & 0 & 0 \\
0 & -1 & 0 & 0 \\
0 & 0 & -1 & 0 \\
0 & 0 & 0 & 1
\end{array}\right)
$$


and where relations (3.5) or (3.6) and

$$
\gamma_{4} \gamma_{\lambda} \gamma_{4}=a_{\lambda \alpha} \gamma_{\alpha}
$$

have been used. The quantity in the bracket [ ] is that which enters the conventional Hamiltonian for the beta-decay. Under space-time transformations it is transformed as a four-vector. The whole term $V^{\lambda}$, including the internal fields $\varphi(\alpha)$, also transforms as a four-vector. The left and the right part of the term $V^{\lambda}$ may belong to different particles. In such a case we use a more precise symbol $V_{a b}^{\lambda}$, where subscripts $a, b$ refer to the fields of two different particles. We must admit that fields belong to different parity classes. In the following we study the transformation properties of $V$-type terms under total space inversion.

$$
\begin{gathered}
V^{\lambda} \equiv V_{r, r}^{\lambda} \rightarrow V_{r, r}^{\prime \lambda}=V^{\prime \lambda} \\
V^{\lambda} \equiv V_{i, i}^{\lambda} \rightarrow V_{i, i}^{\prime \lambda}=V^{\prime \lambda} \\
V_{r, i}^{\lambda} \rightarrow V_{r, i}^{\prime \lambda}=i \varphi_{r}^{\dagger}(\alpha)\left[a_{\lambda \alpha} \bar{\psi}_{r}(x) \gamma_{\alpha} \psi_{i}(x)\right] \varphi_{i}(\alpha)=i a_{\lambda \alpha} V_{r, i}^{\alpha}=i V^{\prime \lambda} \\
V_{i, r}^{\lambda} \rightarrow V_{i, r}^{\prime \lambda}=-\varphi_{i}^{\dagger}(\alpha)\left[a_{\lambda \alpha} \bar{\psi}_{i}(x) \gamma_{\alpha} \psi_{r}(x)\right] \varphi_{r}(\alpha)=-a_{\lambda \alpha} V_{i, r}^{\alpha}=-i V^{\prime \lambda}
\end{gathered}
$$

Indices $r$ and $i$ denote the real and imaginary parity class respectively. The symbol $V$ without subscript stands for the condition where two fields belong to the same parity class.

The other term forms that forms the Hamitloniann is

$$
\begin{aligned}
A^{\lambda}=i \varphi^{\dagger}\left[\bar{\psi}(x) \gamma_{\lambda} \gamma_{5} \psi(x)\right] \varphi(\alpha) \rightarrow A^{\prime \lambda}=i \varphi^{\prime \dagger}\left(\alpha^{\prime}\right)\left[\bar{\psi}^{\prime}\left(x^{\prime}\right) \gamma_{\lambda} \gamma_{5} \psi^{\prime}\left(x^{\prime}\right)\right] \varphi^{\prime}\left(\alpha^{\prime}\right) \\
=i \varphi^{\dagger}(\alpha) P_{I}^{\dagger}\left[\psi^{\dagger}(x) P_{E}^{\dagger} \gamma_{4} \gamma_{\lambda} \gamma_{5} P_{E} \psi(x)\right] P_{I} \varphi_{I}(\alpha) \\
=i \varphi^{\dagger}(\alpha)\left[a_{\lambda \alpha} \bar{\psi}(x) \gamma_{\alpha} P_{E}^{-1} \gamma_{5} P_{E} \psi(x)\right] \varphi(\alpha) \\
=-i \varphi^{\dagger}(\alpha)\left[a_{\lambda \alpha} \bar{\psi}(x) \gamma_{\alpha} \gamma_{5} \psi(x)\right] \varphi(\alpha) \\
=-a_{\lambda \alpha} A^{\alpha}
\end{aligned}
$$

where relations (3.5) or (3.6), (3.7) and

$$
\gamma_{4} \gamma_{5} \gamma_{4}=-\gamma_{5}
$$

have been used. The term $A^{\lambda}$ transforms as an axial vector. The mixed quantities transform as

$$
\begin{gathered}
A_{r, r}^{\lambda} \rightarrow A_{r, r}^{\prime \lambda}=A^{\prime \lambda} \\
A_{i, i}^{\lambda} \rightarrow A_{i, i}^{\prime \lambda}=A^{\prime \lambda} \\
A_{r, i} \rightarrow A_{r, i}^{\prime}=i a_{\lambda \alpha} A^{\alpha}=i A^{\prime \lambda} \\
A_{i, r} \rightarrow A^{\prime}{ }_{i, r}=-i a_{\lambda \alpha} A^{\alpha}=-i A^{\prime \lambda}
\end{gathered}
$$

The Hamiltonian $H_{\text {int }}$ can be rewritten in a compact notation,

$$
H_{\mathrm{int}}=\frac{G_{V}}{\sqrt{2}}\left(V_{p, n}^{\lambda} V_{e, \nu}^{\lambda}+\frac{\eta}{i} V_{p, n}^{\lambda} A_{e, \nu}^{\lambda}\right)+\frac{G_{A}}{\sqrt{2}}\left(A_{p, n}^{\lambda} A_{e, \nu}^{\lambda}+i \eta A_{p, n}^{\lambda} V_{e, \nu}^{\lambda}\right)
$$


This Hamiltonian is invariant under total space inversion, i.e.,

$$
H_{\mathrm{int}}=H_{\mathrm{int}}^{\prime}=H_{\mathrm{int}}
$$

if particles are distributed into parity classes in the following way:

\begin{tabular}{llll}
$p$ & $n$ & $e$ & $\nu$ \\
\hline$r$ & $r$ & $r$ & $r$ \\
$i$ & $i$ & $i$ & $i$ \\
$r$ & $r$ & $i$ & $i$ \\
$i$ & $i$ & $r$ & $r$ \\
$r$ & $i$ & $i$ & $r$ \\
$i$ & $r$ & $r$ & $i$ \\
\hline
\end{tabular}

This can be verified directly from the form of the Hamiltonian (3.10) by having in mind the relations (3.8), (3.9) and the relations

$$
\begin{gathered}
\eta^{\prime}=-\eta \\
V_{p, n}^{\prime \lambda} V_{e, \nu}^{\prime \lambda}=\left(a_{\lambda \alpha} V_{p, n}^{\alpha}\right)\left(a_{\lambda \beta} V_{e, \nu}^{\beta}\right)=V_{p, n}^{\lambda} V_{e, \nu}^{\lambda} \\
V_{p, n}^{\prime \lambda} A_{e, \nu}^{\prime \lambda}=-V_{p, n}^{\lambda} A_{e, \nu}^{\lambda} \\
A_{p, n}^{\prime \lambda} V_{e, \nu}^{\prime \lambda}=-A_{p, n}^{\lambda} V_{e, \nu}^{\lambda} \\
A_{p, n}^{\prime \lambda} A_{e, \nu}^{\prime \lambda}=A_{p, n}^{\lambda} A_{e, \nu}^{\lambda}
\end{gathered}
$$

Thus we have constructed the Hamiltonian for the beta-decay that is invariant under total space inversion. The old Hamiltonian for the beta-decay (Lee \& Yang, 1957 ) is only a special case of this more general Hamiltonian. If we apply only the external space inversion $P_{E}$, then $\eta^{\prime}=\eta$, and $H_{\text {int }}$ obviously changes its form Our Hamiltonian is for the ordinary particles $P_{+}, n_{+}, \nu_{+}$, the same as the conventional one (except for the additional $\phi^{\dagger}(\alpha)$ and $\varphi(\alpha)$ ), because all these particles have $\alpha=1$, and therefor $\eta=+1$. It does not conserve parity.

The main objection against the scheme of Yang and Tiomno (Yang \& Tiomno, 1950), namely that it fails in the case of beta-decay, because the weak interaction is not invariant under space inversion, is thus surmounted. This forgotten scheme explains the conservation of baryons by assuming that baryons and leptons belong to two different parity classes and, further, that all interactions are invariant under space inversion. Then, indeed, interactions containing terms which create (annihilate) a baryon and simultaneously annihilate (create) a lepton would be excluded, because under inversion a factor $i$ would appear. However, this scheme does not explain the separate conservation of electron leptons and myon leptons. Perhaps this additional complication could be explained by taking into account the internal degrees of freedom. For our present purpose it is not necessary to propose any detailed scheme. We wish to show only new theoretical possibilities, connected with a formal existence of two parity classes. 
We now return to the main subject, the explanation of beta-decay in terms of a Hamiltonian that is invariant under total space inversion. We shall no longer distinguish between real and imaginary parity class, so it is reasonable to assume that all particles belong to the real class - according to usual practice.

If in the initial state there is a positron $e_{+}^{+}$and neutron $n_{+}$, the Hamiltonian described by equation (3.10) produces the process

$$
e_{+}^{+}+n_{+} \rightarrow p_{+}^{+}+\bar{\nu}_{+}(\uparrow)
$$

The symbol $(\uparrow)$ denotes the positive helicity $(\mathbf{s} \cdot \mathbf{p}>0)$ and the symbol $(\downarrow)$ the negative helicity $(\mathbf{s} \cdot \mathbf{p}<0)$. A process obtained from (3.11) by external space inversion

$$
e_{+}^{+}+n_{+} \rightarrow p_{+}^{+}+\bar{\nu}_{+}(\downarrow)
$$

does not occur under the action of $H_{\text {int }}$ (equation (3.10)). But according to our hypothesis a process exist which is obtained from (3.11) by application of total space inversion:

$$
e_{-}^{+}+n_{-} \rightarrow p_{-}^{+}+\bar{\nu}_{-}(\downarrow)
$$

This last process is governed by the same Hamiltonian (3.10) as process (3.11).

Let us sum up. The Hamiltonian $H_{\text {int }}$ is such that the initial state $\left|e_{+}^{+} n_{+}\right\rangle$ develops into the final state $\left|p_{+}^{+} \bar{\nu}_{+}(\uparrow)\right\rangle$ and the initial state $\left|e_{-}^{+} n_{-}\right\rangle$into the final state $\left|p_{-}^{+} \bar{\nu}_{-}(\downarrow)\right\rangle$. The mirror particles $e_{-}^{+}, e_{-}^{-}, \bar{n}_{-}, p_{-}^{+}, p_{-}^{-}, \nu_{-}, \bar{\nu}_{-}$, etc., have all the properties of mass, charge, spin, etc., similar to the corresponding ordinary particles, except that their behavior is different in the processes with weak interaction, they have the opposite internal handedness. Their existence is a logical consequence of the postulate that each physical theory must be invariant under total space inversion. The theory of beta-decay, formulated with the aid of mirror particles, is indeed invariant under total space inversion. Mention should be made that the sufficient condition for the invariance is the existence of mirror nucleons. The existence of mirror leptons is not necessary for reflection invariance.

\section{Discussion and Conclusion}

We have shown that non-conservation of parity in beta-decay does not necessarily imply the mirror asymmetry of basic laws of nature. If we take into account the structure of nucleons we can imagine that this structure is asymmetric with respect to space inversion and, because of this fact, the angular distribution of particles at the beta-decay is also asymmetric. In other words, what is asymmetric at the beta-decay are initial conditions, while the weak interaction itself is symmetric. In order to formulate this idea more precisely we have made a distinction among external, internal and total space inversion. The space inversion is said to be total if applied to all degrees of freedom that we are able to control by experiment, at least in principle. Whilst discussing reflection invariance or non-invariance of the beta-decay, conventionally we have in mind the external space-inversion, and 
not the total one, because we have not mentioned the inversion of the internal structure of the nucleon. In the present paper we have shown that by including the internal structure of particles involved in the beta-decay we can in principle restore reflection invariance. Of course, our proposal must be confirmed expermentally. But in any case, the conclusion that the non-conservation of parity - the external parity - means that nature is not invariant under space inversion is wrong. Nonconservation of external parity means only that nature is not invariant under partial space inversion, but reveals nothing about invariance or non-invariance under total space inversion.

In the present work we have postulated that nature is invariant under total space inversion. This assumption is justified by the fact that nature has always appeared to be symmetric in its basic laws. On the other hand, the exact validity of dynamical symmetries has proved many times in history to be only an extrapolation. For instance, the conservation of mechanical energy is only an idealization which holds in the absence of dissipative forces. One could have said in the early stages of the development of physics that the invariance under a translation in time is only approximative. However, by including the internal translational and rotational degrees of freedom (which manifest themselves in the heat movement of molecules) the conservation od energy is again restored. But in the next step one can measure precisely the kinetic energies of molecules and observe that energy is not strictly conserved. Firstly because of inelastic atomic excitations and, secondly, because of the equivalence of mass and energy. Next, the conservation of energy was seriously questioned in the beta-decay before the discovery of neutrino. At every stage one could question the invariance of Lagrangian theories under a translation in time, but such has not been the behavior of physicists. The strong belief in time translation invariance forced them to correct their theories, that have at last been confirmed by experiment. In the case of reflection invariance the situation is similar. Geometrical space inversion is only an idealization, and how it could be applied as an active transformation on a dynamical system which has yet unknown structure in the microdomain cannot be imagined. In the present work we have seen, in a very rough manner, how the theory of beta-decay can be formulated to be invariant under space inversion. This can be achieved by formally including in the description the structure of nucleons.

\section{Acknowledgement}

I thank Prof. Dr. J. Strnad, and Dr. M. Vakselj for very useful discussions.

References

Fraunfelder, H.R. et al. (1957). Physical Review, 106, 386.

Lee, T.D. and Yang, C.N. (1957). Physical Review, 105, 1671

Muirhead, H. (1965)). The Physics of Elementary Particles. Pergamon Press Ltd.

Salam, A. (1957). Nuovo Cimento, 5, 299.

Yang, C.N. and Tiomno, J. (1950). Physical Review, 79, 498. 